\title{
Brownian pump powered by a white-noise flashing ratchet
}

\author{
A. Gomez-Marin and J. M. Sancho \\ Facultat de Fisica, Universitat de Barcelona, Diagonal 647, 08028 Barcelona, Spain
}

(Received 6 September 2007; published 7 March 2008)

\begin{abstract}
A Brownian pump of particles powered by a stochastic flashing ratchet mechanism is studied. The pumping device is embedded in a finite region and bounded by particle reservoirs. In the steady state, we exactly calculate the spatial density profile, the concentration ratio between both reservoirs and the particle flux. We propose a simulation framework for the consistent evaluation of such observable quantities.
\end{abstract}

DOI: 10.1103/PhysRevE.77.031108

PACS number(s): 05.40.-a, 05.70.Ln

\section{INTRODUCTION}

Free diffusion by itself is not the appropriate physical mechanism for selective transport processes. The appearance of net directed motion needs the breaking of detailed balance and spatial inversion symmetry. Inspired on Feynman's ratchet and pawl device [1], such phenomenon has been named after the ratchet effect. Moreover, when the environment is thermally fluctuating, Brownian transport is crucially affected and controlled by noise (for a broad review, see [2] and references therein). Its relevance to molecular machines, such as motors, pumps, and channels, has been proposed from the foundation idea of the so-called Brownian motors [3].

Brownian pumping is then an active nonequilibrium transport process in which fluctuations play a very important role. Regarding experiments, Na,K-ATPase pumps have been perturbed by an oscillating electric field [4,5], driving ions whose net flux was measured as a function of the amplitude and frequency of the field. The flux of particles created by pumping devices has been studied theoretically in [6-9]. Generally, a typical ratchet mechanism is assumed with periodic boundary conditions for the (normalized) probability distribution of particles, putting the emphasis on the calculation of the flux as a function of the model parameters. In this work, the scenario is different and, at the same time, more suitable to model real pumps. While the ratchet mechanism is still responsible for the transport of particles, we focus on the nonequilibrium concentration gradient created and maintained by the pumping device. Thus our system is not periodic (see Fig. 1). We consider the unnormalized density of particles at the boundaries as the main observable quantity. The flux $J$ of particles, together with the density profile $\rho(x)$ in the membrane and the ratio of concentrations $\rho_{1} / \rho_{0}$ originated between the particle reservoirs, are studied.

The structure of paper is the following. First we introduce the model for the Brownian pump by means of a Langevin equation, which can be mapped into a Seebeck ratchet [10-12]. In the steady state, a standard theoretical analysis is carried out to obtain the nonequilibrium density of particles $\rho(x)$ that is generated at zero and finite flux. The flux $J$ itself is also determined. Then we present a simple numerical scheme from which the densities and fluxes can be measured and satisfactorily compared to the predictions. For a piecewise linear saw-tooth potential we calculate explicitly the expressions derived in the analysis and explore them as a function of the main parameters of the system. We end with some conclusions and comments for future work.

\section{GENERAL THEORETICAL ANALYSIS}

We consider an underdamped Brownian particle moving on a line under a time dependent potential $V(x, t)$. The corresponding equation of motion for its position is the Langevin equation

$$
m \ddot{x}=-\gamma \dot{x}-V^{\prime}(x, t)+\eta(t),
$$

where $m$ is the mass of the particle, $\gamma$ introduces the friction, and $\eta(t)$ is a Gaussian white noise accounting for thermal fluctuations of the environment with the usual autocorrelation

$$
\left\langle\eta(t) \eta\left(t^{\prime}\right)\right\rangle=2 \gamma k_{B} T \delta\left(t-t^{\prime}\right) .
$$

The time dependent potential $V(x, t)$ consists of a ratchet part $V(x)$ (a spatially asymmetric potential) time modulated by a stochastic process in the following form:

$$
V(x, t)=V(x)[1+\chi(t)],
$$

where $\chi(t)$ is another Gaussian white noise with zero mean, uncorrelated with $\eta(t)$, whose autocorrelation is

$$
\left\langle\chi(t) \chi\left(t^{\prime}\right)\right\rangle=2 Q \delta\left(t-t^{\prime}\right) .
$$

In the regime in which friction dominates inertia, the Langevin equation reduces to the so-called overdamped limit,

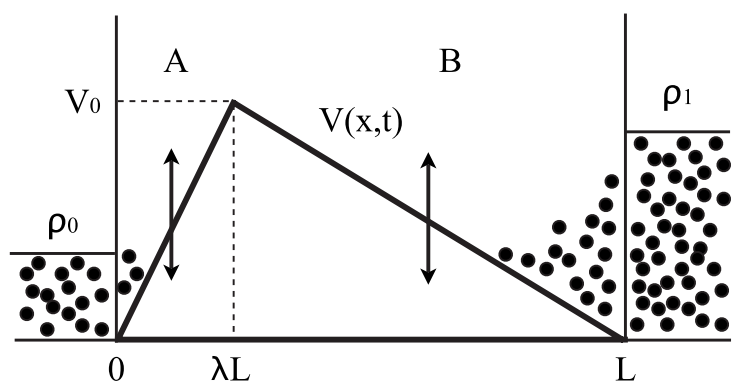

FIG. 1. Scheme of the pumping device: A spatially asymmetric and time dependent potential $V(x, t)$, embedded in a finite region of length $L$ (denoted as the membrane) flashes in time creating a density profile between the reservoirs of densities $\rho_{0}$ and $\rho_{1}$. 


$$
\dot{x}=-V^{\prime}(x)-V^{\prime}(x) \chi(t)+\eta(t),
$$

where the friction constant $\gamma$ has been set to one.

It is worth remarking here that, due to the inertia term, the Ito and Stratonovich interpretations of Eqs. (1)-(4) coincide despite being a stochastic differential equation with multiplicative noise. Equation (5), however, should be treated with care, since one must decipher first whether it has to be interpreted according to either Ito or Stratonovich rules. The order of the limiting procedures from which one arrives to an overdamped equation from an underdamped equation, having assumed first that the noise $\chi(t)$ is obtained as the limit of an Orstein-Ulhenbeck process, determines that the appropriate stochastic interpretation is that of Ito [13-15]. Then, Eq. (5) can be rewritten as

$$
\dot{x}=-V^{\prime}(x)+g(x) \xi(t),
$$

where

$$
g(x)=\sqrt{k_{B} T+Q V^{\prime}(x)^{2}},
$$

and the new effective noise $\xi(t)$ has zero mean and correlation $\left\langle\xi(t) \xi\left(t^{\prime}\right)\right\rangle=2 \delta\left(t-t^{\prime}\right)$. The continuity equation for the density of particles $\rho(x, t)$ is

$$
\partial_{t} \rho(x, t)=-\partial_{x} J(x, t) .
$$

By using Ito's prescription in Eq. (6), the expression for the flux $J(x, t)$ yields [16]

$$
-J(x, t)=V^{\prime}(x) \rho(x, t)+\partial_{x}\left[g^{2}(x) \rho(x, t)\right] .
$$

Although Eqs. (8) and (9) correspond to the well known Fokker-Planck equation, here $\rho(x, t)$ accounts for the physical density of particles and not the usual (normalized) probability distribution.

In the steady state the density $\rho(x)$ is just a function of space and thus the flux $J$ becomes a constant. The density follows a first order nonhomogeneous linear differential equation whose formal solution is

$$
\rho(x)=\mathcal{Z}\left(x, c_{0}, J\right) \exp \left[-\int_{0}^{x} d z\left(\frac{V^{\prime}(z)}{g^{2}(z)}+2 \frac{g^{\prime}(z)}{g(z)}\right)\right],
$$

with

$$
\mathcal{Z}\left(x, c_{0}, J\right)=c_{0}-J \int_{0}^{x} \frac{d z}{g^{2}(z)} \exp \left(\int_{0}^{z} d y\left[\frac{V^{\prime}(y)}{g^{2}(y)}+2 \frac{g^{\prime}(y)}{g(y)}\right]\right) .
$$

The unknown constant $c_{0}$ is found by imposing the left reservoir concentration, $\rho_{0} \equiv \rho\left(0^{-}\right)$, as a fixed boundary condition. Then $c_{0}=\rho_{0}$. At each boundary between the membrane and the reservoirs (at $x=0$ and $x=L$ ) we will distinguish between approaching from the left and the right side due to possible discontinuities in the derivatives of the potential.

In what follows, we will study two different situations. First, we impose a zero total flux: $J=0$. This corresponds to the case in which the pump is maintaining the maximum concentration difference between the two reservoirs across the membrane with no net leaking of particles. This situation is analogous to the stalling force in Brownian motors. From Eq. (10), the density profile in the membrane is

$$
\rho(x)=\rho\left(x_{0}\right)\left[\frac{g\left(x_{0}\right)}{g(x)}\right]^{2} \exp \left[-\int_{x_{0}}^{x} \frac{V^{\prime}(z)}{g^{2}(z)} d z\right] .
$$

The exponent 2 in the prefactor before the exponential is a characteristic of Ito's interpretation. It changes to 1 for Stratonovich's. One can find the expression for the ratio of concentrations at both sides of the pumping device by defining $\rho_{1} \equiv \rho\left(L^{+}\right)$. Assuming no systematic drift in the system, this is $V^{\prime}\left(L^{+}\right)=V^{\prime}\left(0^{-}\right)$; then $g\left(L^{+}\right)=g\left(0^{-}\right)$and therefore Eq. (12) yields

$$
\frac{\rho_{1}}{\rho_{0}}=\exp \left[-\frac{1}{k_{B} T} \int_{0^{-}}^{L^{+}} \frac{V^{\prime}(z)}{1+\frac{Q}{k_{B} T} V^{\prime}(z)^{2}} d z\right]
$$

The concentration $\rho_{0}$ was fixed in the former case. If we now impose $\rho_{1}$ too, then the flux $J$ is no longer zero in general. From Eqs. (10) and (11), one can get the explicit expression

$$
J=\frac{\rho_{0}-\rho_{1} \exp \left(\int_{0^{-}}^{L^{+}} d z\left[\frac{V^{\prime}(z)}{g^{2}(z)}+2 \frac{g^{\prime}(z)}{g(z)}\right]\right)}{\int_{0^{-}}^{L^{+}} \frac{d z}{g^{2}(z)} \exp \left(\int_{0^{-}}^{z} d y\left[\frac{V^{\prime}(y)}{g^{2}(y)}+2 \frac{g^{\prime}(y)}{g(y)}\right]\right)} .
$$

Substituting $J$ back in Eq. (11) one can obtain, from Eq. (10), the steady density $\rho(x)$ for any choice of $\rho_{0}$ and $\rho_{1}$.

These two last equations are the main analytical results of this paper. They will be explicitly evaluated for the piecewise linear potential of Fig. 1 and compared with numerical simulations.

\section{SIMULATION FRAMEWORK}

Let us suppose that the analytical solutions for the density and flux derived in the previous sections are not available. Then one may need to think of a simulation framework to numerically obtain those relevant observables for pumps and channels. A possible option is to solve numerically the differential equation for the density $\rho(x)$ with appropriate boundary conditions. However, a different and much more interesting perspective consists in simulating the dynamics of many noninteracting particles (described by the Langevin equation) in the membrane and the reservoirs and, afterward, extracting the concentration profiles directly from their positions. This approach is much closer to real experiments in biomembranes. The present section is devoted to describing in detail such a numerical scheme.

There are multiple choices for a stochastic algorithm to simulate the particle dynamic equations (5) or (6). The simplest and good enough for the case under consideration is the first order Euler's algorithm. For Eq. (6), the position $x$ at time $t+\delta t$ of the $i$ th particle turns out to be

$$
x_{i}(t+\delta t)=x_{i}(t)-V^{\prime}\left[x_{i}(t)\right] \delta t+g\left[x_{i}(t)\right] X_{i}(t),
$$

where $\delta t$ is the (small) integration time step and the stochastic term $X_{i}(t)$ is constructed as, 


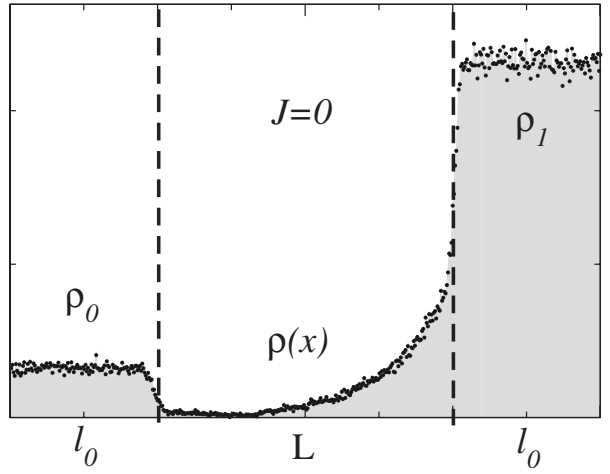

FIG. 2. Histogram of the density profile $\rho(x)$ in the steady state at $J=0$ obtained from numerical simulations. We use the potential introduced in Sec. IV. Reflecting boundary conditions are implemented at the end of both reservoirs.

$$
X_{i}(t)=\int_{t}^{t+\delta t} \xi\left(t^{\prime}\right) d t^{\prime}=\sqrt{2 \delta t} \alpha_{i}
$$

in which $\alpha_{i}$ are independent Gaussian random numbers $N(0,1)$. The algorithm used in Eq. (15) corresponds to Ito's interpretation for a general model.

The main feature of the simulation framework we present is the implementation of the boundary conditions that account for the particle reservoirs at both sides of the membrane. Using an auxiliary length $l_{0}$, we extend and divide the spatial domain of the system into three regions: The left reservoir in $x \in\left[-l_{0}, 0\right)$, the membrane in $x \in(0, L)$, and the right reservoir in $x \in\left(L, L+l_{0}\right]$. We consider $N$ noninteracting particles, each one evolving according to Eq. (15). When a particle is in a reservoir no deterministic force acts on it. It diffuses freely; only random kicks due to thermal noise drive the particle. The numerical simulation starts with particles distributed randomly in the entire domain. Each particle is numbered and its position, $x_{i}(t)$, is stored. First we observe a transient regime during which $\rho_{0}(t)$ decreases and $\rho_{1}(t)$ increases, up to the steady state in which $\rho_{1}>\rho_{0}$ remains constant. Once this stage is reached, the density profile $\rho(x)$ is obtained as a histogram of the spatial distribution of particles. The following typical values of the simulation dimensionless parameters are used: $L=1, l_{0}=0.5, \delta t=0.001$, and $N=500$ and the overall time of observation in the steady state is of the order of $t=1000$. Notice that one can lower such time by increasing the number of particles (or vice versa) keeping the statistics constant since, from the ergodicity hypothesis, ensemble averages are equal to time averages.

The two possible situations, $J=0$ and $J \neq 0$, need a different approach. For the first case $(J=0)$, we impose reflecting boundary conditions at the end points $\left(x=-l_{0}\right.$ and $\left.x=L+l_{0}\right)$. This means that if at any integration step the particle crosses the end points, we restore its position with the specular image with respect to such a boundary. As shown in Fig. 2, the steady state density of particles at both reservoirs is constant. Moreover, from this simple histogram of the positions of the particles, the density profile is obtained.

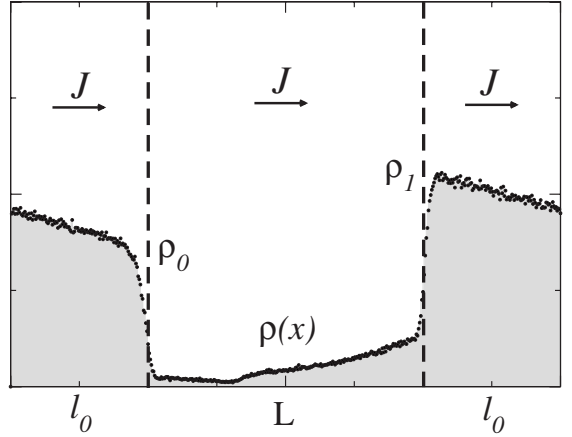

FIG. 3. Histogram of the density profile $\rho(x)$ in the steady state at $J \neq 0$ obtained from numerical simulations. Periodic boundary conditions are applied at the ends of the reservoirs leading naturally to a constant flux $J$ everywhere and fixed $\rho_{1}$ and $\rho_{0}$.

For the second case $(J \neq 0)$, we impose periodic boundary conditions at points $-l_{0}$ and $L+l_{0}$, so that both end points are connected. At the steady state, $\rho_{0} \neq \rho_{1}$ is observed, with a linear density profile in the reservoirs which is the signature of a finite nonzero constant flux $J$. See Fig. 3. More advanced and sophisticated studies of simulation of Langevin trajectories with specific boundary conditions can be found in Refs. [17-19]. In our method, the pump is moving particles from the left to the right forcing a concentration gradient in the reservoirs (which are connected only in the numerical scheme). Accordingly, a net flux appears that fulfills Fick's law,

$$
J=\frac{k_{B} T}{2 l_{0}}\left(\rho_{1}-\rho_{0}\right) .
$$

This simple and intuitive method is able to generate data of $J, \rho_{0}$, and $\rho_{1}$ since, independent of the initial conditions, the system will evolve to the steady state in which the value of $J$ in Eqs. (14) and (17) coincides. Then the flux can be measured either by counting the net number of particles crossing the point $x=L+l_{0}$ (where the periodic condition is implemented) as a function of time or from the histogram of $\rho(x)$ on the region $\left(L, L+l_{0}\right) \cup\left(-l_{0}, 0\right)$ by fitting Eq. (17). From this the concentrations $\rho_{0}$ and $\rho_{1}$ can be determined as well. Regarding the effect that the discontinuous forces have on the accuracy of the simulations, one can see that at the interfaces of the reservoir and the membrane the density quickly falls, trying to mimic jumps in $\rho(x)$ that come from the piecewise linear potential. The abrupt changes observed indicate that the time-step is small enough to faithfully reproduce the effect of such force discontinuities (see details in Fig. 4).

On the whole, the above explained rules and conditions lead to the physical situation of interest. The quantitative agreement between theory predictions and simulation results is good as we will show in the next section.

\section{ANALYTICAL AND SIMULATION RESULTS}

In this section we complete the preliminary analytical results of Ref. [20] and compare them with numerical simula- 


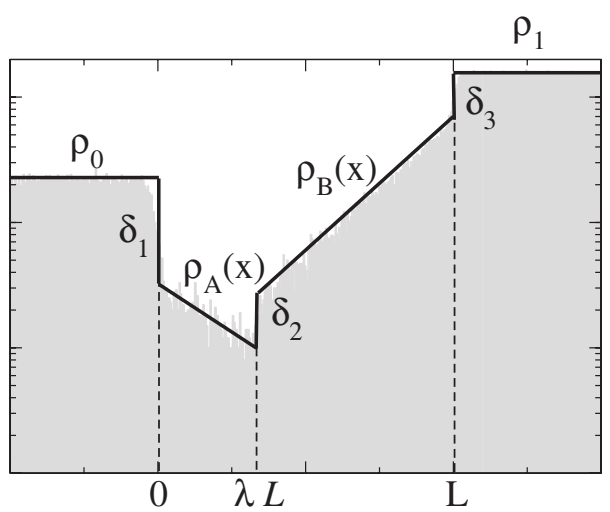

FIG. 4. Density profile $\rho(x)$ for $v_{0}=8, \lambda=1 / 3$, and $\alpha=0.01$ in logarithmic scale. The histogram from simulations (in gray) falls just on top of the theoretical predictions (solid lines).

tions. The explicit model we consider is a piecewise linear saw-tooth potential depicted in Fig. 1. It is defined in two regions as

$$
\begin{gathered}
V_{A}(x)=V_{0} \frac{x}{\lambda L}, \quad x \in(0, \lambda L), \\
V_{B}(x)=V_{0} \frac{L-x}{(1-\lambda) L}, \quad x \in(\lambda L, L) .
\end{gathered}
$$

$V_{0}$ is the height of the potential, $\lambda$ (which can only take values between 0 and 1) controls the asymmetry, and $L$ is the total length where the pumping device is allocated.

The forthcoming subsections are devoted to calculating and discussing the exact analytical expressions of the profile density of particles $\rho(x)$ and the concentration ratio $\rho_{1} / \rho_{0}$ at $J=0$, as well as the normalized flux $J / \rho_{0}$ as a function of $\rho_{1} / \rho_{0}$. Since the potential is piecewise linear, the force is discontinuous, yielding to discontinuities in the density profiles, which we will carefully characterize without any further problems.

\section{A. Density profile $\rho(x)$ at $J=0$}

Let us recall expression (12) for the spatial density profile $\rho(x)$ given a baseline concentration $\rho_{0}$ and zero flux conditions $J=0$. From the potential in Eqs. (18) and (19), $\rho(x)$ can be obtained exactly. Nevertheless, as the potential is piecewise linear, we expect discontinuities in $\rho(x)$. Thus we have to evaluate the profile in four different zones denoted by the subscripts $0, A, B$, and 1, whose meaning is clear from Figs. 1 and 4 . First we introduce the following dimensionless parameters:

$$
v_{0} \equiv \frac{V_{0}}{k_{B} T}, \quad \alpha \equiv \frac{Q k_{B} T}{L^{2}},
$$

where $v_{0}$ is the relative energy barrier of the potential compared to the thermal energy. The parameter $\alpha$ is a measure of the strength of the flashing mechanism.

The density profile of particles in region $A$, this is $\rho_{A}(x)$, in which $x \in\left(0^{+}, \lambda L^{-}\right)$, is obtained from Eq. (12) by taking $x_{0}=0^{-}$, so that $\rho\left(x_{0}\right)=\rho_{0}$ and $g\left(x_{0}\right)=\sqrt{k_{B} T}$. Noticing that $g(x)$ in region $A$ [denoted by $g_{A}(x)$ ] is a constant, one has

$$
g_{A}=g\left(0^{+}\right)=g\left(\lambda L^{-}\right)=\sqrt{k_{B} T+Q\left(V_{0} / \lambda L\right)^{2}},
$$

which finally yields to

$$
\rho_{A}(x)=\frac{\rho_{0}}{\omega_{1}} \exp \left(-\frac{v_{0}}{\omega_{1}} \frac{x}{\lambda L}\right),
$$

where, for simplicity in the notation, the following new dimensionless quantities have been defined:

$$
\omega_{1} \equiv 1+\alpha\left(\frac{v_{0}}{\lambda}\right)^{2}, \quad \omega_{2} \equiv 1+\alpha\left(\frac{v_{0}}{1-\lambda}\right)^{2} .
$$

Then the jump of the concentration at $x=0$ is simply

$$
\delta_{1} \equiv \rho\left(0^{-}\right)-\rho\left(0^{+}\right)=\rho_{0}\left(1-\frac{1}{\omega_{1}}\right) .
$$

The density of particles in region $B$ is found similarly. We recall Eq. (12) and now choose $x_{0}=\lambda L^{-}$. From the above expressions and by noticing again that $g_{B}(x)$ is a constant,

$$
g_{B}=\sqrt{k_{B} T+Q\left[V_{0} /(1-\lambda) L\right]^{2}},
$$

the concentration profile $\rho_{B}(x)$ is found to be

$$
\rho_{B}(x)=\frac{\rho_{0}}{\omega_{2}} \exp \left(-\frac{v_{0}}{\omega_{1}}\right) \exp \left(\frac{v_{0}}{\omega_{2}} \frac{x-\lambda L}{L-\lambda L}\right) .
$$

The jump between zones $A$ and $B$ (at $x=\lambda L$ ) is

$$
\begin{aligned}
\delta_{2} & \equiv \rho\left(\lambda L^{+}\right)-\rho\left(\lambda L^{-}\right)=\rho_{B}(\lambda L)-\rho_{A}(\lambda L) \\
& =\rho_{0} \exp \left(-\frac{v_{0}}{\omega_{1}}\right)\left(\frac{1}{\omega_{2}}-\frac{1}{\omega_{1}}\right) .
\end{aligned}
$$

The constant density $\rho_{1}$ at the other side of the membrane is discussed in detail in the next section. The jump $\delta_{3}$, which corresponds to $x=L$, is obtained likewise from the difference between $\rho_{1}$ and $\rho_{B}(L)$. In Fig. 4 we show, in logarithmic scale, the above analytical predictions for $\rho(x)$ calculated in every region and the corresponding jumps. Note that every piece of prediction fits perfectly to the histogram (in gray) built from the simulation data.

\section{B. Ratio $\rho_{1} / \rho_{0}$ at $J=0$}

We focus now on the value of the ratio of concentrations at both ends of the membrane that the pumping Brownian device is able to create and maintain at the stalling regime. For the linear saw-tooth potential, Eq. (13) gives

$$
\frac{\rho_{1}}{\rho_{0}}=\exp \left[v_{0}\left(\frac{1}{1+\alpha\left(\frac{v_{0}}{1-\lambda}\right)^{2}}-\frac{1}{1+\alpha\left(\frac{v_{0}}{\lambda}\right)^{2}}\right)\right] .
$$

Let us explore the dependence of the above expression on the parameters $v_{0}, \alpha$, and $\lambda$. In Fig. 5 we check the symmetry properties of our prediction with respect to the parameter $\lambda$. This figure shows the right-left inversion symmetry of the problem when we change $\lambda$ for $1-\lambda$. Note that at $\lambda=0.5$, although there is a time modulation of the potential, the de- 


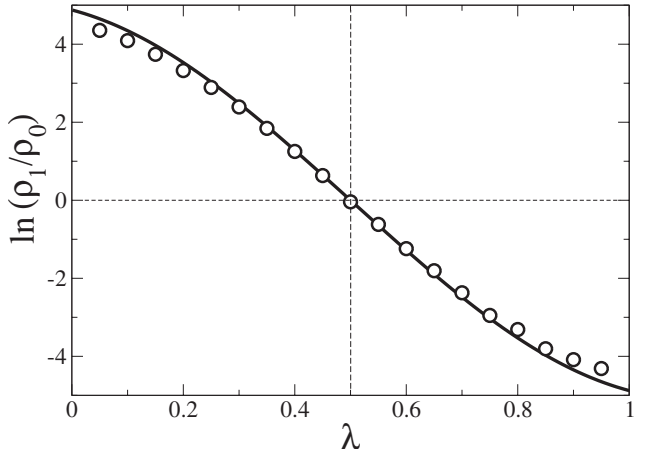

FIG. 5. Logarithm of the ratio of concentrations vs the asymmetry parameter $\lambda$ at $J=0$. Note that it is antisymmetric under the transformation $\lambda \leftrightarrow 1-\lambda$. The parameters are $v_{0}=8$ and $\alpha=0.01$. Solid line corresponds to theory, Eq. (28), and circles to simulation data.

vice does not pump because the spatial inversion symmetry is not broken and so there is no preferred direction. The more asymmetric the potential, the greater the pumping capacity achieved. This does not mean higher efficiencies with respect to energy consumption. This issue is not studied in this work.

In Fig. 6 the density ratio is studied versus the relative energetic barrier $v_{0}$. There is an optimum value which gives the largest difference. If the barrier height is small with respect the thermal energy, diffusive losses through the pump are important and the ratio decreases. Note that for $v_{0} \rightarrow 0$ there is no concentration difference; $\rho_{1}=\rho_{0}$. However, for very large values of $v_{0}$, few particles can cross the barrier and get to the other side despite the flashing of the potential. The pumping decreases again.

In Fig. 7 we show the ratio of concentrations versus $\alpha$ (we have varied $Q$ in the $\alpha$ exploration). For low values of $Q$, the potential barely changes in time, diffusion dominates, and thus particles can scarcely be pumped. For a very strong flashing the potential is so often distorted that particles do not have time to cross through the membrane and they are again poorly pumped. In between both regimes, there is a region that enhances transport. Such an optimal regime indicates that the flashing intensity $Q$ can be tuned to be optimal. This is a common feature of flashing ratchets [2]. The maximum appearing in Figs. 6 and 7 can be compared qualita-

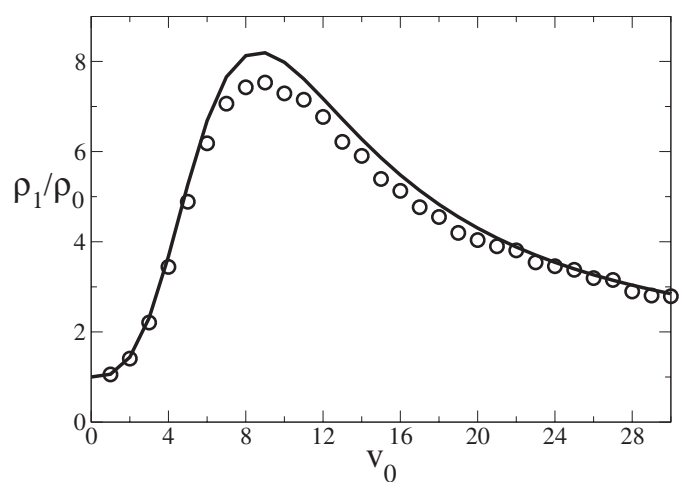

FIG. 6. Ratio of concentrations $\rho_{1} / \rho_{0}$ as a function of the relative potential barrier $v_{0}$ at $J=0$. The values of the parameters are $\lambda=1 / 3$ and $\alpha=0.01$. Line and circles as in previous figure.

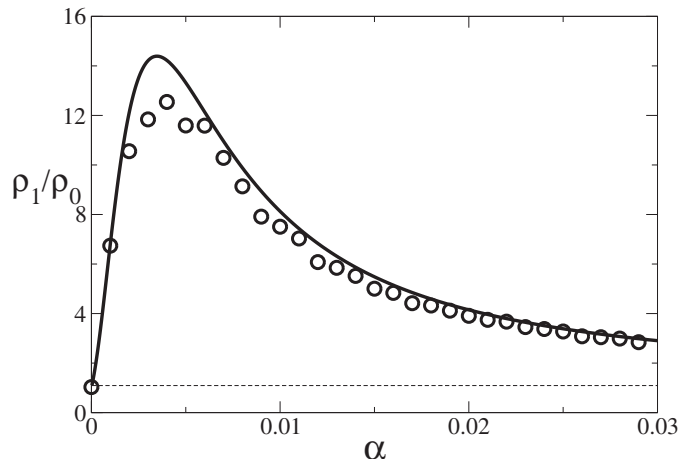

FIG. 7. Ratio of concentrations vs parameter $\alpha$ at $J=0$ for $v_{0}$ $=8$ and $\lambda=1 / 3$. Line and circles as in previous figure.

tively with the experimental results of $[4,5]$ for the amplitude and frequency of the flashing perturbation. Let us analyze in more detail the case $\alpha=0$, which gives $\rho_{0}=\rho_{1}$. This limit is physically interesting because it corresponds to $Q k_{B} T / L^{2}$ $\rightarrow 0$. When the intensity in the multiplicative noise vanishes $(Q=0)$, the breaking of detailed balance does not occur and so the ratchet effect cannot take place. Moreover, another way to make $\alpha$ vanish is to set $T=0$. We have to be very careful because $T$ also appears in $v_{0}=V_{0} / k_{B} T$. In fact, in the absence of thermal fluctuations, the flashing ratchet mechanism still works because the multiplicative noise does all the whole job (breaks detailed balance and supplies fluctuations). Therefore, in the $\alpha$ exploration we have kept $T$ different from zero.

\section{Flux $J / \rho_{0}$ versus ratio $\rho_{1} / \rho_{0}$}

We can recall expression (14) for the total flux of particles and rewrite it as

$$
\frac{J}{\rho_{0}}=\frac{1-\rho_{1} / \rho_{1}^{\text {stall }}}{\frac{1}{k_{B} T} \int_{0^{-}}^{L^{+}} d z \exp \left(\int_{0^{-}}^{z} d y \frac{V^{\prime}(y)}{g^{2}(y)}\right)},
$$

where $\rho_{1}^{\text {stall }}$ is the concentration in the right reservoir when $J=0$, which is calculated in Eq. (13). For the linear saw-tooth potential, the above formal expression can be explicitly expressed as

$$
J=\left(\frac{k_{B} T}{L}\right) \frac{\rho_{0} e^{-v_{0} / \omega_{1}}-\rho_{1} e^{-v_{0} / \omega_{2}}}{\mathcal{N}_{1}+\mathcal{N}_{2}},
$$

where

$$
\begin{gathered}
\mathcal{N}_{1}=\frac{\omega_{1}}{v_{0}} \lambda\left(1-e^{-v_{0} / \omega_{1}}\right), \\
\mathcal{N}_{2}=\frac{\omega_{2}}{v_{0}}(1-\lambda)\left(1-e^{-v_{0} / \omega_{2}}\right) .
\end{gathered}
$$

Expression (30) fulfills the symmetry $J \rightarrow-J$ when $\lambda \leftrightarrow 1$ $-\lambda$ and $\rho_{0} \leftrightarrow \rho_{1}$, which means that if we take the mirror image of the setup, we should see the same flux going to the opposite direction. Note that when $J=0$ only the ratio of 


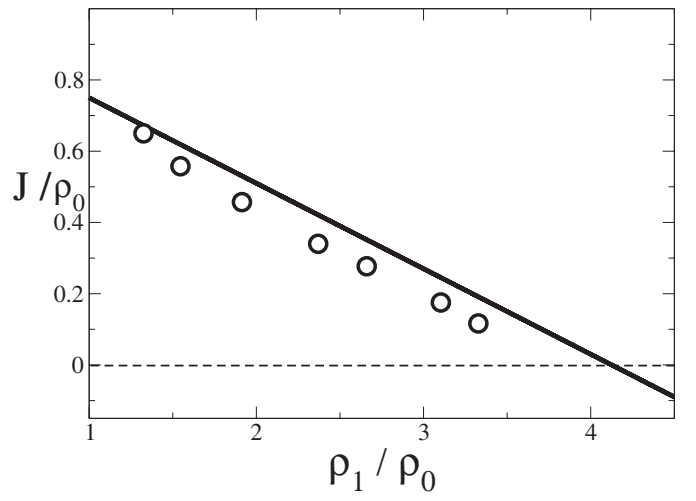

FIG. 8. Flux $J / \rho_{0}$ as a function of $\rho_{1} / \rho_{0}$. The system parameters are $\lambda=1 / 3, v_{0}=8, \alpha=0.02$, and $L=1$. Solid line corresponds to theory, Eq. (30), and circles to simulation data.

densities is relevant, while, for $J \neq 0$, both values are needed separately. There is a decreasing linear behavior of $J$ with respect to $\rho_{1}$, as it is clear from simple inspection of Eqs. (29) and (30). If $\rho_{1}>\rho_{1}^{\text {stall }}$ the flux is reversed because entropic forces surmount the pumping driving.

In Fig. 8 the normalized flux $J / \rho_{0}$ is plotted as a function of the density ratio $\rho_{1} / \rho_{0}$. The simulation points are successfully obtained from the method explained in the numerical scheme section. Increasing the length $l_{0}$ of the left and right reservoirs, the value of the flux $J$ decreases. We can measure it from the slope of the linear profile of the density in such regions and also extract $\rho_{0}$ and $\rho_{1}$. Despite some small deviations due to the finite statistics and finite $\delta t$, we can say that theory and simulations are in agreement. This confirms the validity of the numerical scheme proposed, which allows us to measure fluxes and concentrations from simple numerical simulations which have a clear physical interpretation.

\section{CONCLUSIONS}

Brownian pumps, unlike typical Brownian motors, do not aim to create a net flux of particles in periodic boundary conditions but, instead, to achieve and actively keep a density gradient between two reservoirs. As a first approach to theoretically study such devices from this perspective, we have considered a simple but physically relevant model based on the ratchet effect with appropriate boundary conditions. It has been characterized and explored from the theoretical point of view with analytical exact results. Moreover, a simulation framework for obtaining the concentration and the flux predicted theoretically has been proposed. Instead of numerically solving the differential equation for the density, we simulate a Langevin dynamics of independent particles. Albeit the algorithm itself is standard, the suitable implementation of boundary conditions in every case $(J=0$ and $J \neq 0)$ leads naturally to a direct identification of the histogram of the positions of the particles with the density profile. This does not require one to insert and remove particles as they cross the interface that separates the membrane from the particle reservoirs. The numerical scheme presented can be of utility in those cases in which analytical predictions are not possible.

The efficiency of pumps is not easy to investigate since one should analyze how much energy the fluctuating potential is inserting into the pump and what is the energetic profit taken out from such input. This issue together with the use of parameter values in the biological scale is under study. The present work is then a starting point for modeling nanometric biomachines, such as channels and pumps, which control the flux of particles across the cell membrane.

\section{ACKNOWLEDGMENTS}

We are indebted to J. Casademunt for fruitful discussions on diffusion subtleties. We acknowledge financial help from the Ministerio de Educacion y Ciencia (Spain) under Project No. FIS2006-11452-C03-01 and Grant No. FPU-AP-20040770 (A.G.-M.).
[1] R. P. Feynman, R. B. Leighton, and M. Sands, The Feynman Lectures on Physics (Addison Wesley, Reading, MA, 1963), Vol. 1, pp. 46.1-46.9.

[2] P. Reimann, Phys. Rep. 361, 57 (2002).

[3] G. Oster, Nature (London) 417, 25 (2002).

[4] D. S. Liu, R. D. Astumian, and T. Y. Tsong, J. Biochem. (Tokyo) 265, 7260 (1990).

[5] T. Y. Tsong and T. D. Xie, Appl. Phys. A: Mater. Sci. Process. 75, 345 (2002).

[6] J. Prost, J.-F. Chauwin, L. Peliti, and A. Ajdari, Phys. Rev. Lett. 72, 2652 (1994).

[7] R. D. Astumian and I. Derenyi, Phys. Rev. Lett. 86, 3859 (2001).

[8] R. D. Astumian, Phys. Rev. Lett. 91, 118102 (2003).

[9] I. Kosztin and K. Schulten, Phys. Rev. Lett. 93, 238102 (2004).

[10] M. Buttiker, Z. Phys. B: Condens. Matter 68, 161 (1987).

[11] Y. M. Blanter and M. Buttiker, Phys. Rev. Lett. 81, 4040 (1998).
[12] A. Gomez-Marin and J. M. Sancho, Phys. Rev. E 71, 021101 (2005).

[13] J. M. Sancho, M. San Miguel, and D. Durr, J. Stat. Phys. 28, 291 (1982).

[14] J. M. Sancho and A. Sanchez, Eur. Phys. J. B 16, 127 (2000).

[15] R. Kupferman, G. A. Pavliotis, and A. M. Stuart, Phys. Rev. E 70, 036120 (2004).

[16] H. Risken, The Fokker-Planck Equation, Springer Series in Synergetics (Springer, Berlin, 1984), Vol. 18.

[17] B. Nadler, Z. Schuss, and A. Singer, Phys. Rev. Lett. 94, 218101 (2005).

[18] R. S. Eisenberg, M. M. Klosek, and Z. Schuss, J. Chem. Phys. 102, 1767 (1995).

[19] P. Szymczak and A. J. C. Ladd, Phys. Rev. E 69, 036704 (2004).

[20] J. M. Sancho and A. Gomez-Marin, in Noise and Fluctuations in Biological, Biophysical, and Biomedical Systems, Proceedings of SPIE, edited by S. M. Bezrukov (SPIE, Bellingham, WA, 2007), Vol. 6602, p. 6602OB. 